\title{
Lemnalosides A-D, Decalin-type Bicyclic Diterpene Glycosides from the Marine Soft Coral Lemnalia sp.
}

Guangmin Yao, ${ }^{\dagger}{ }^{\ddagger}$ Nicholas B. Vidor, ${ }^{\dagger}$ Adam P. Foss ${ }^{\dagger}$, and Leng Chee Chang ${ }^{\dagger, *}$ Department of Chemistry and Biochemistry, College of Science and Engineering, University of Minnesota Duluth, 1039 University Drive, Duluth, MN 55812.

\section{List of Supporting Information}

Figure S1. ${ }^{1} \mathrm{H}$ NMR spectrum of Lemnaloside A (1; $\left.500 \mathrm{MHz}, \mathrm{CDCl}\right) \quad$ P2

Figure S2. ${ }^{13} \mathrm{C}$ NMR spectrum of LemnalosideA (1; $\left.125 \mathrm{MHz}, \mathrm{CDCl} 3\right) \quad$ P3

Figure S3. ${ }^{1} \mathrm{H}$ NMR spectrum of Lemnaloside B (2; $\left.500 \mathrm{MHz}, \mathrm{CDCl}\right) \quad$ P4

Figure S4. ${ }^{13} \mathrm{C}$ NMR spectrum of Lemnaloside B (2; $\left.125 \mathrm{MHz}, \mathrm{CDCl} 3\right) \quad$ P5

Figure S5. ${ }^{1} \mathrm{H}$ NMR spectrum of Lemnaloside C (3; $300 \mathrm{MHz}$, Acetone-d6) P6

Figure S6. ${ }^{13} \mathrm{C}$ NMR spectrum of Lemnaloside C (3; $100 \mathrm{MHz}$, Acetone-d6) $\quad$ P7

Figure S7. ${ }^{1} \mathrm{H}$ NMR spectrum of Lemnaloside D (4; $\left.500 \mathrm{MHz}, \mathrm{CDCl}\right) \quad$ P8

Figure S8. ${ }^{13} \mathrm{C}$ NMR spectrum of Lemnaloside E (4; $\left.125 \mathrm{MHz}, \mathrm{CDCl} 3\right) \quad$ P9

\footnotetext{
${ }^{*}$ To whom correspondence should be addressed. Tel: 218-726-7346 Fax: 218-726-7394.

E-mail: lcchang@d.umn.edu

${ }^{\dagger}$ University of Minnesota Duluth.

‡ Shijiazhuang Pharmaceutical Group Zhongqi (sjz) Pharmaceutical Technology Co., Ltd.
} 
Figure S1. ${ }^{1} \mathrm{H}$ NMR spectrum of Lemnaloside A (1; $\left.500 \mathrm{MHz}, \mathrm{CDCl} 3\right)$

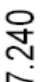

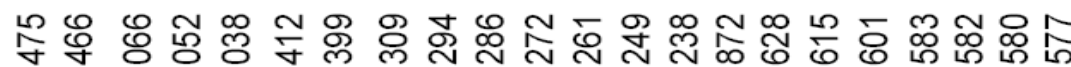

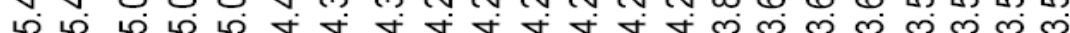

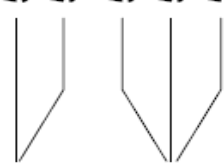

Lemnalosdie A (1) CDC13 1H-NMR

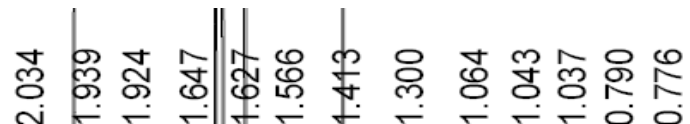

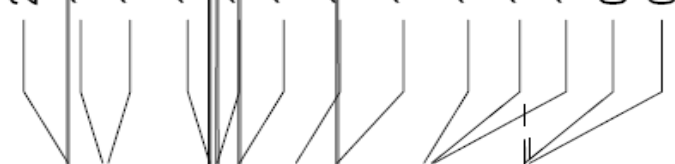

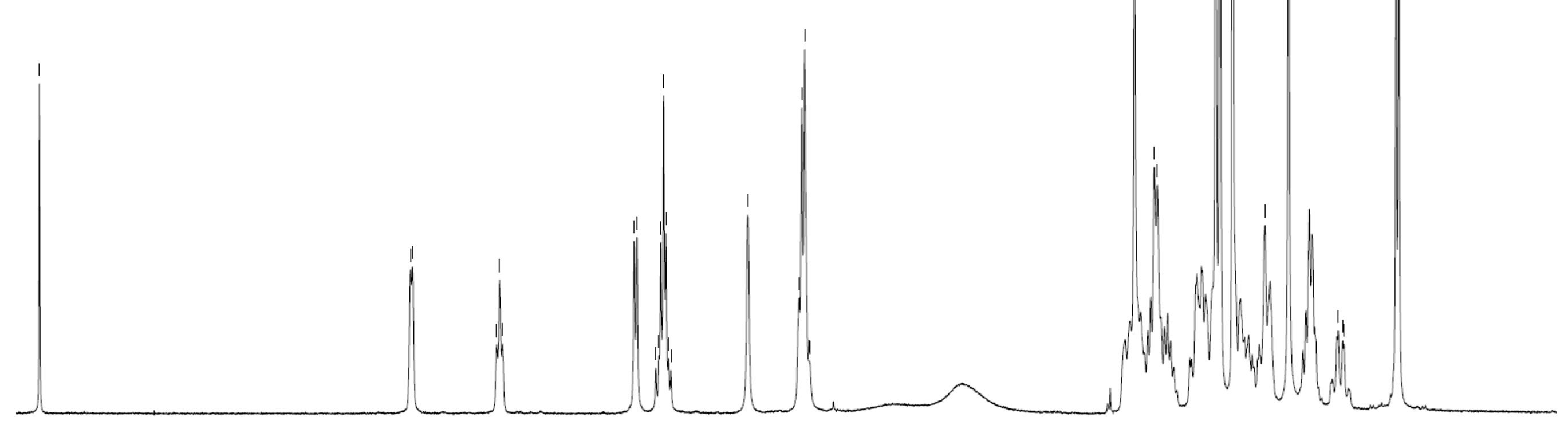

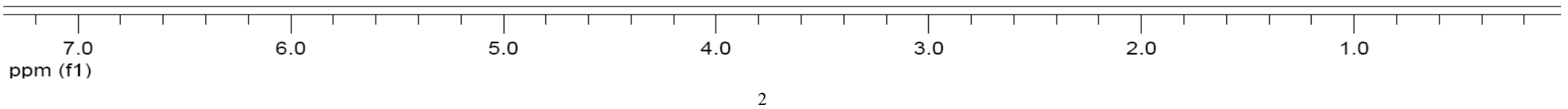


Figure S2. ${ }^{13} \mathrm{C}$ NMR spectrum of LemnalosideA (1; $\left.125 \mathrm{MHz}, \mathrm{CDCl} 3\right)$

을

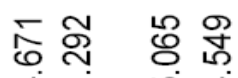

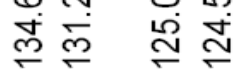

号

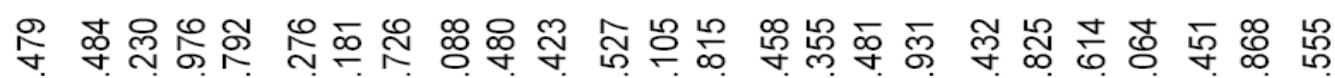

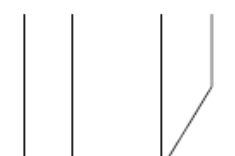

○

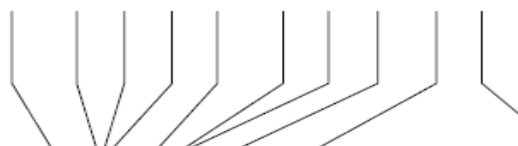

Lemnaloside A (1) CDC13 13C-NMR
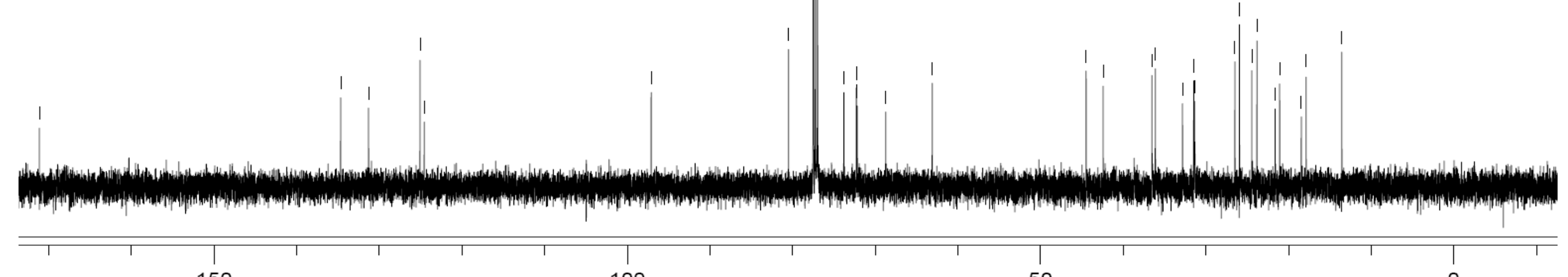
Figure S3. ${ }^{1} \mathrm{H}$ NMR spectrum of Lemnaloside B (2; $\left.500 \mathrm{MHz}, \mathrm{CDCl} 3\right)$ ம ம ம

\section{Lemnaloside B (2) CDCl3 $1 \mathrm{H}-\mathrm{NMR}$}
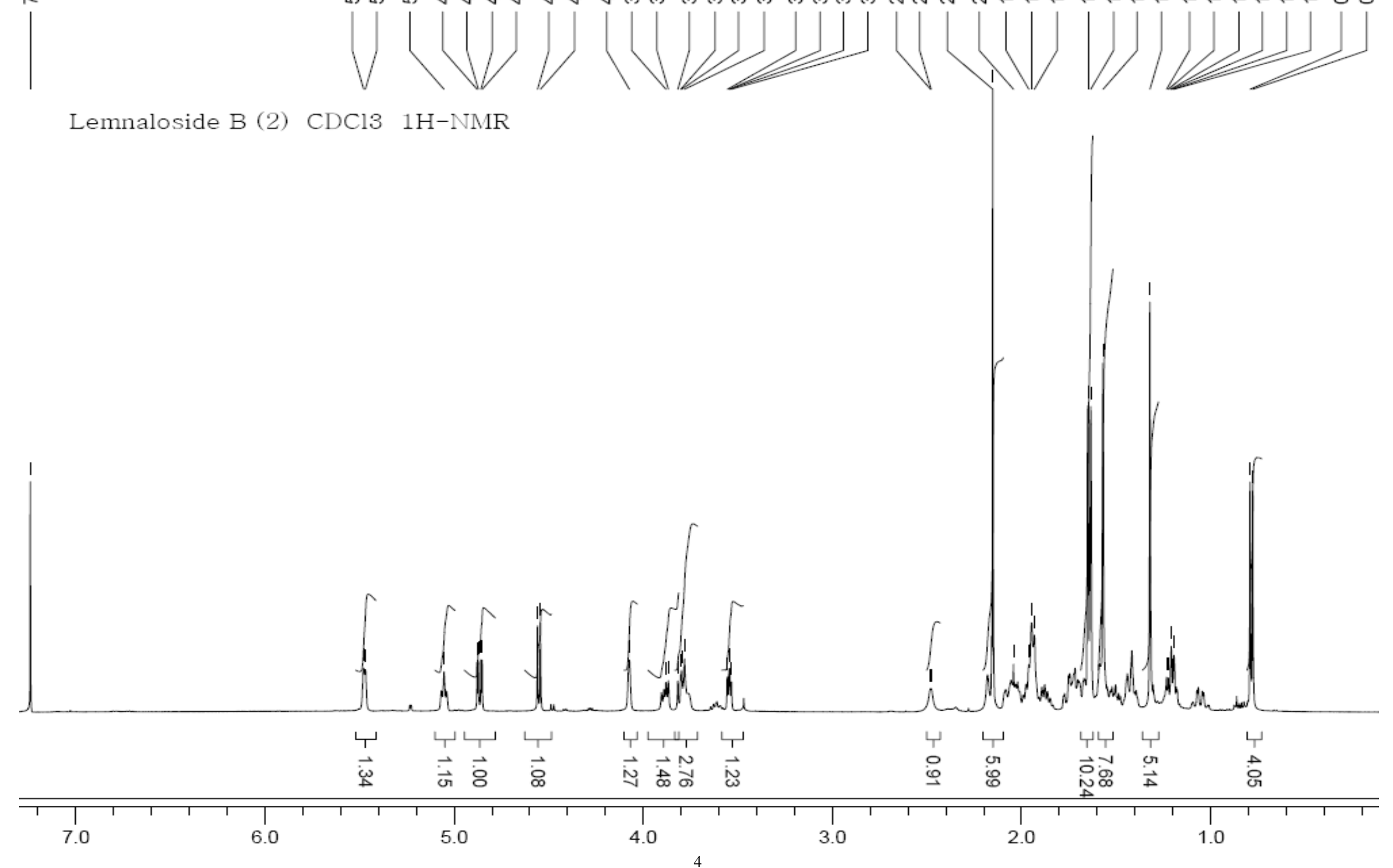
Figure S4. ${ }^{13} \mathrm{C}$ NMR spectrum of Lemnaloside B (2; $\left.125 \mathrm{MHz}, \mathrm{CDCl} 3\right)$

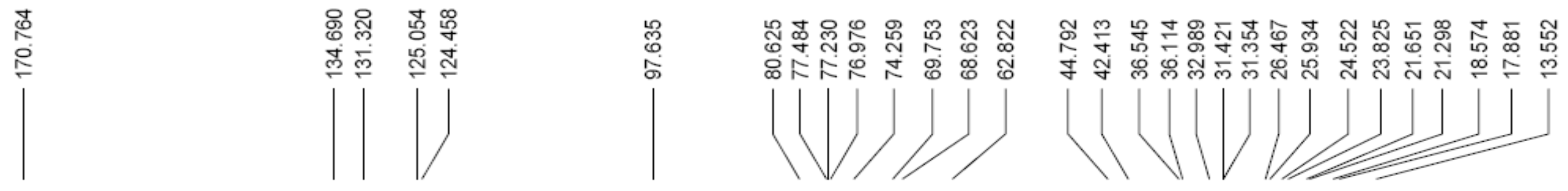

Lemnaloside B (2) CDCl3 13C-NMR

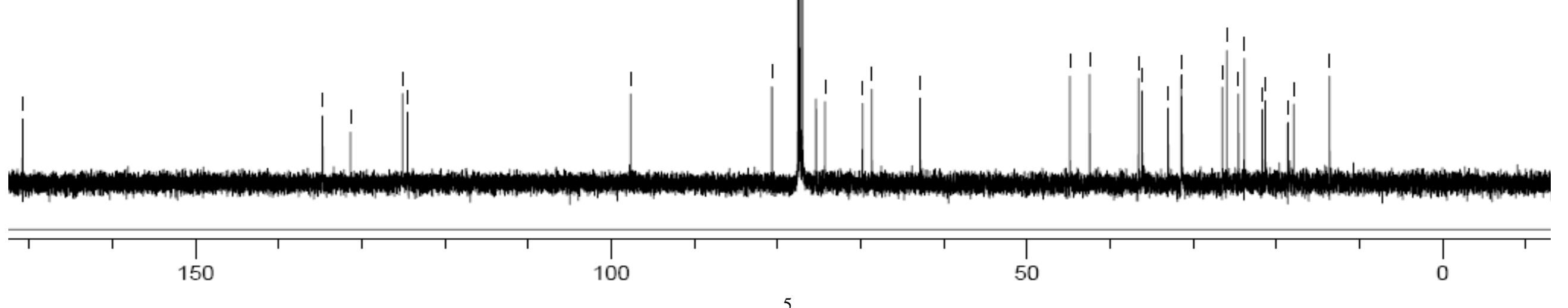


Figure S5. ${ }^{1} \mathrm{H}$ NMR spectrum of Lemnaloside C (3; $300 \mathrm{MHz}$, Acetone-d6)

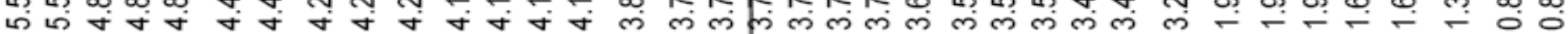

Lemnaloside C (3) $1 \mathrm{H}$ Acetone-d6
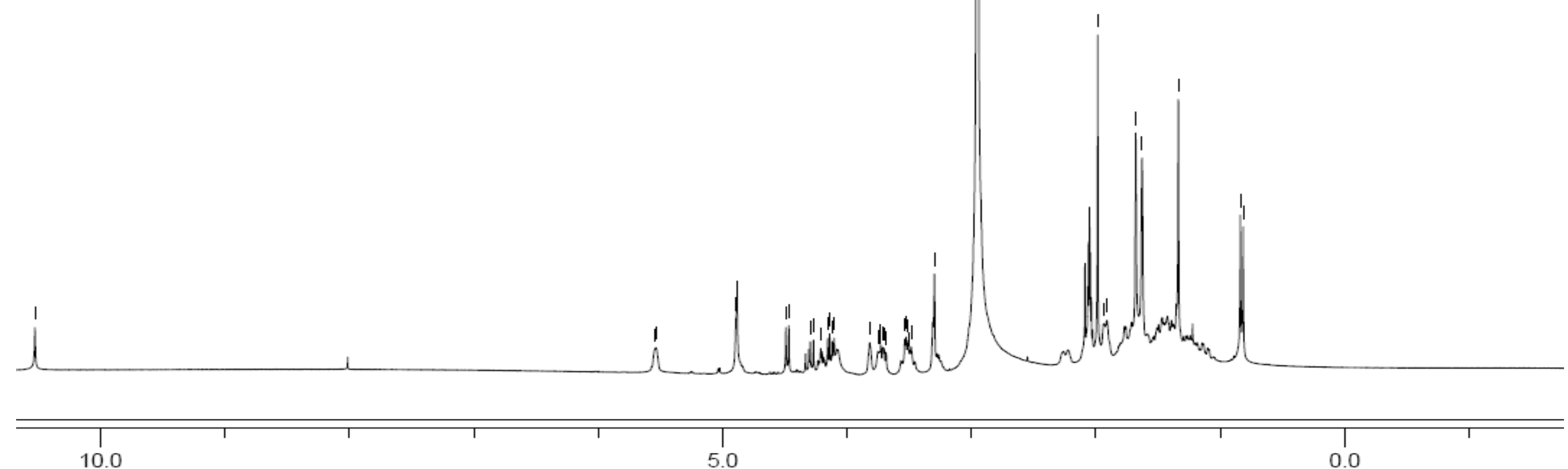


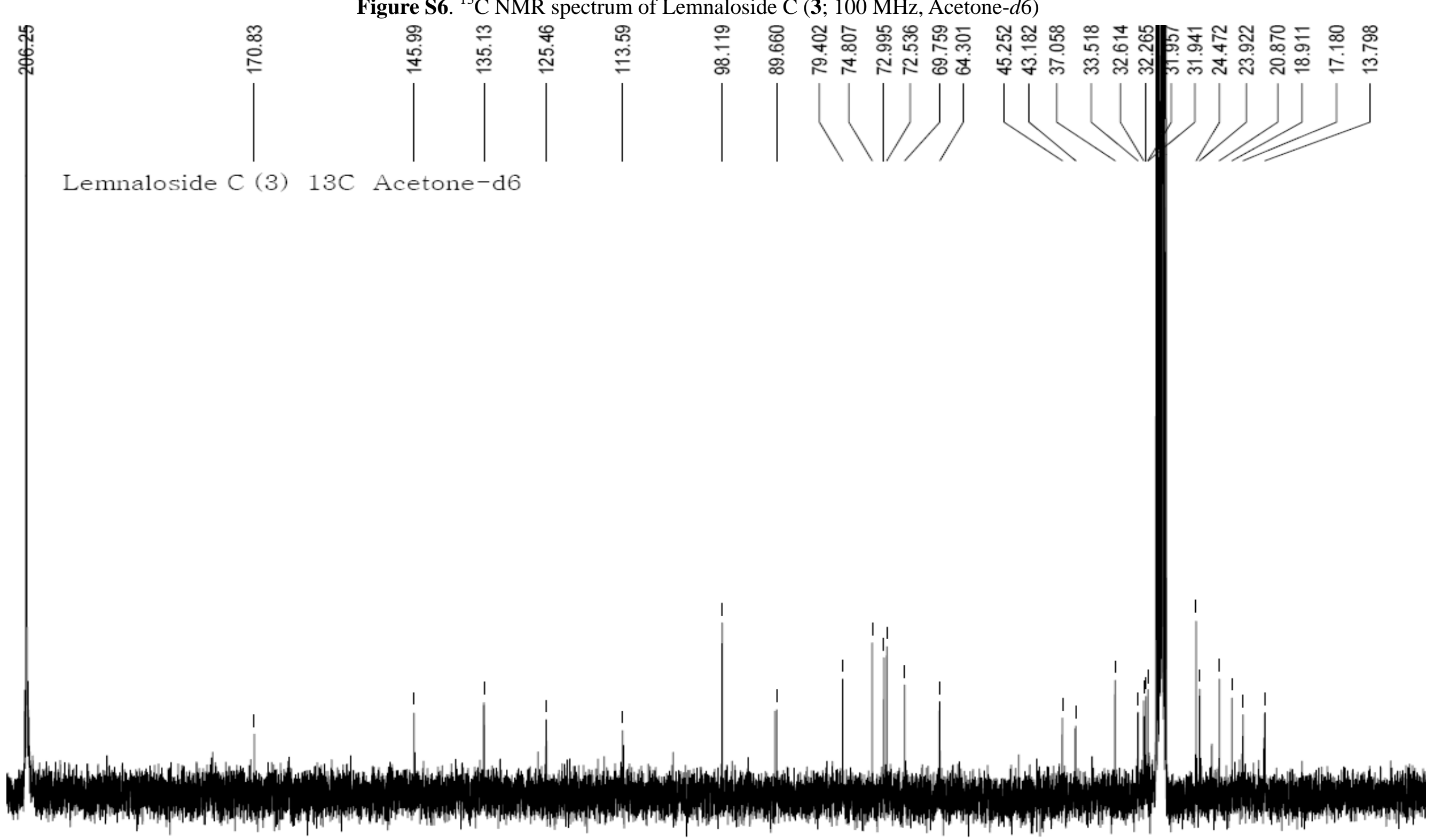

\begin{tabular}{|c|c|c|c|c|}
\hline $\begin{array}{r}\mid \\
200 \\
\mathrm{ppm}(\mathrm{t} 1)\end{array}$ & $\begin{array}{c}1 \\
150\end{array}$ & $\begin{array}{c}1 \\
100\end{array}$ & $\begin{array}{c}1 \\
50\end{array}$ & $\begin{array}{l}T_{0} \\
\end{array}$ \\
\hline
\end{tabular}


Figure S7. ${ }^{1} \mathrm{H}$ NMR spectrum of Lemnaloside D (4; $\left.500 \mathrm{MHz}, \mathrm{CDCl} 3\right)$
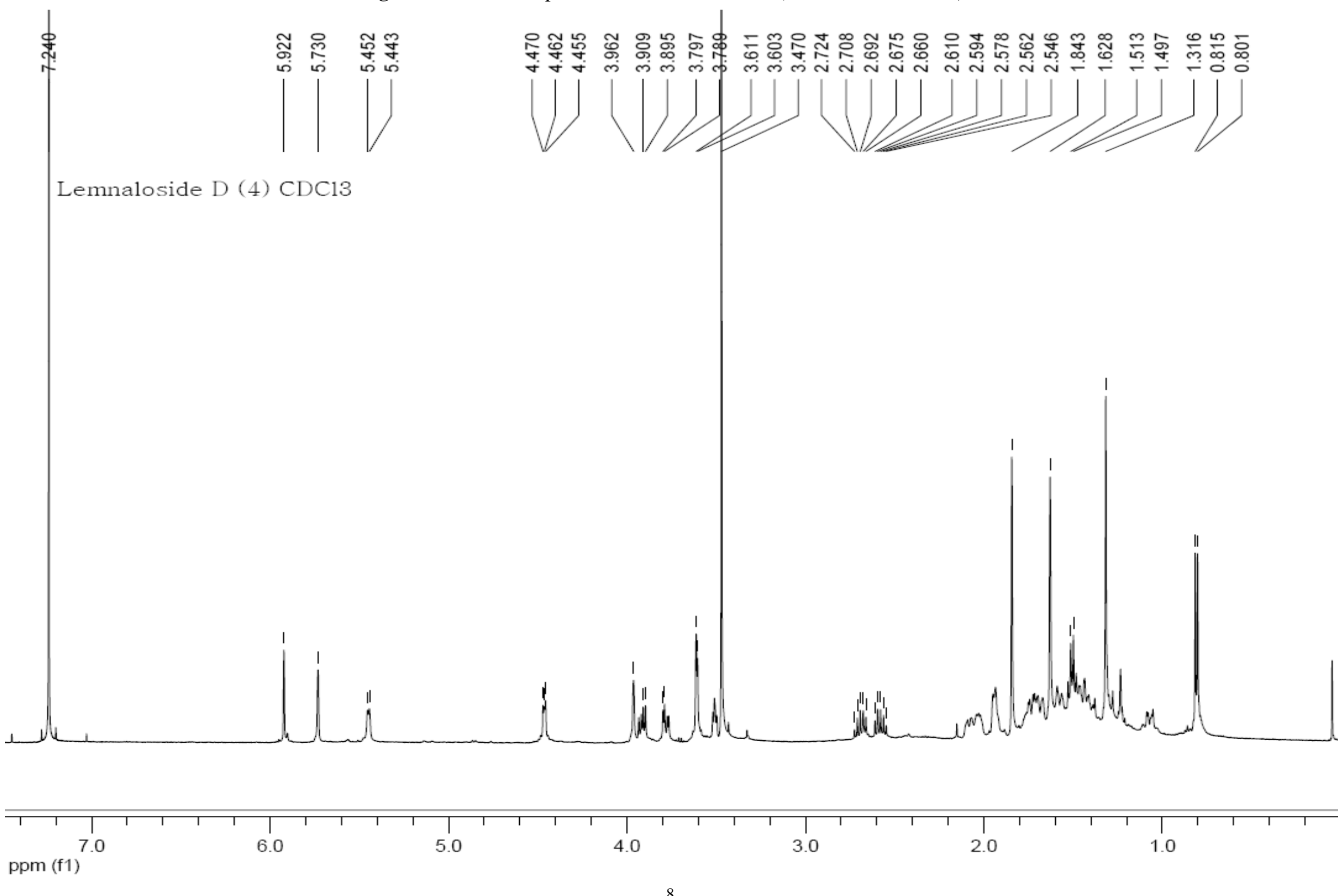
Figure S8. ${ }^{13} \mathrm{C}$ NMR spectrum of Lemnaloside E (4; $\left.125 \mathrm{MHz}, \mathrm{CDCl} 3\right)$

Lemnaloside D (4) $13 \mathrm{C} \mathrm{CDC13}$
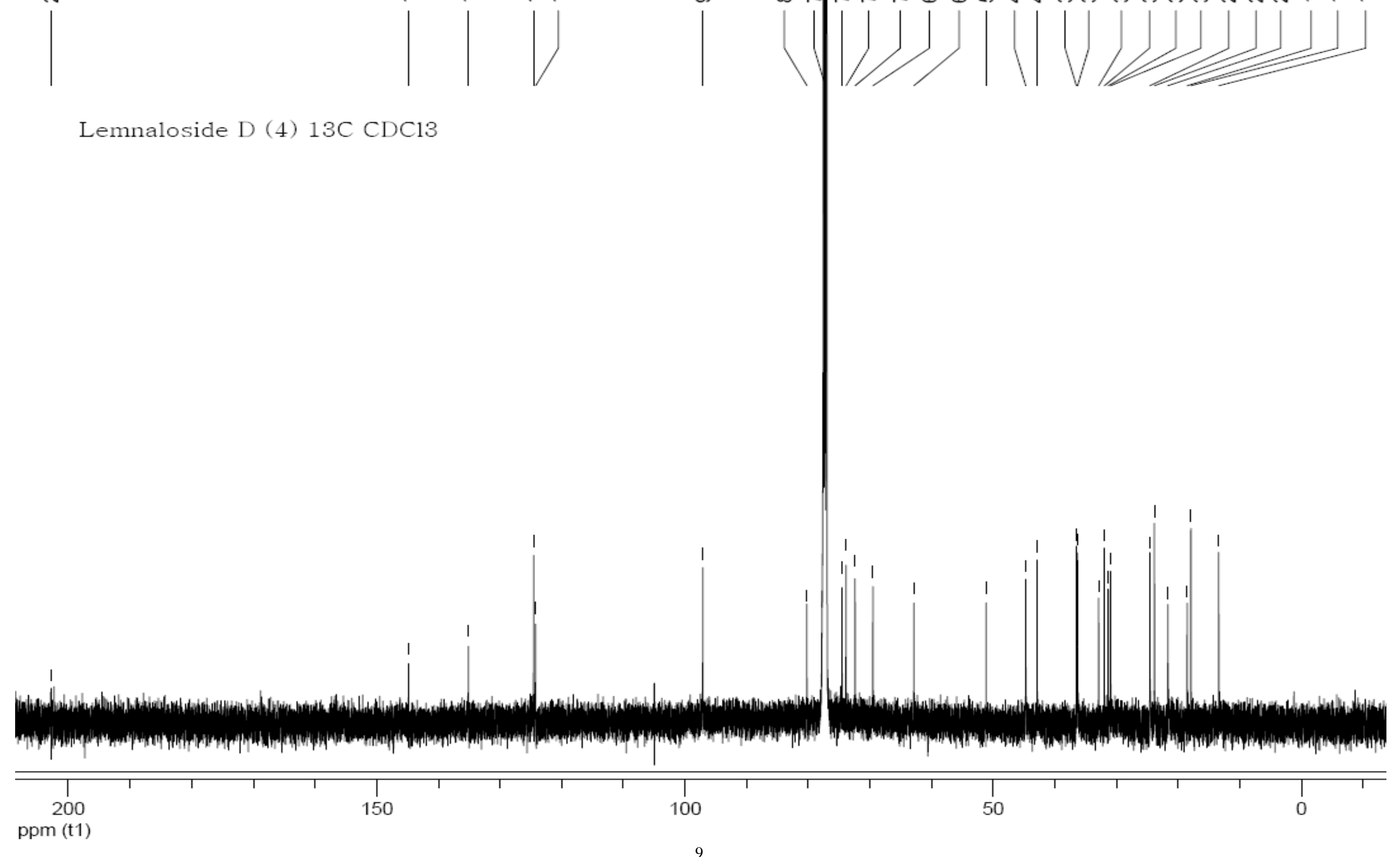